\title{
Simulation Based Design of Sensors
}

Kaltenbacher, Manfred

Applied Mechatronics, Alps-Adriatic University, Klagenfurt, Austria

Corresponding author: Manfred Kaltenbacher, Lakeside B04a, 9020 Klagenfurt, Austria, P: +43(0)463-2700-3562, F: +43(0)463-2700-993568, E: manfred.kaltenbacher@uni-klu.ac.at

Abstract: The design of modern sensors, which are often Micro-Electro-Mechanical Systems (MEMS), strongly depends on the availability of appropriate computer aided engineering (CAE) tools, since the fabrication of each prototype is quite costly. We present the finite network as well as the Finite Element (FE) approach used for the design of a silicon microphone, and how both methods in combination are indispensable tools for an efficient development process.

\section{Introduction}

In most cases, the fabrication of prototypes within the design process of modern sensors is a lengthy and costly task. Therefore, the need for reliable and usable computer modeling tools capable of precisely simulating the multi-field interactions is increased. These computer-aided engineering (CAE) tools offer many possibilities to the design engineer. Arbitrary modification of sensor geometry and selective variation of material parameters are easily performed and the influence on the sensor behavior can be studied immediately. In addition, the simulation provides access to physical quantities that cannot be measured, and simulations strongly support the insight into physical phenomena. Thus, a CAE-based design can tremendously reduce the number of necessary prototypes. However, we want to emphasize that a direct physical control of the sensor design is possible only with the help of experiments, whereas the computer simulation is always based on a model of reality. Therefore, the quality of the results depends on the suitability of the physical model as well as the material parameters [6].

In an early state of the design process it is often advantageously to base the modeling for the numerical simulation on a system of ordinary differential equations (ODEs), which uses finite network elements [1]. Therewith, the engineer can check, if the design goals can be roughly achieved.

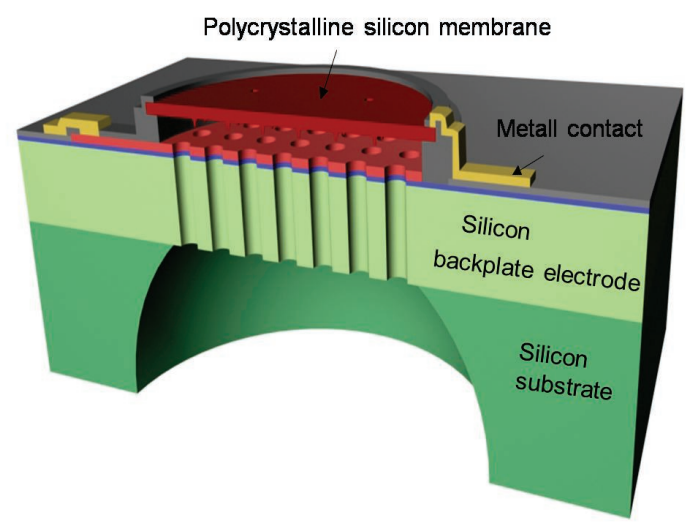

Figure 1: Principle setup of the investigated silicon microphone [3]. 
In the second step, one needs mostly a very accurate model, which will result in a system of coupled partial differential equations (PDEs), which are mainly solved by applying the Finite Element (FE) method [4].

Within this contribution, we will concentrate on the simulation based development of a silicon microphone as displayed in Fig. 1.

\section{Finite Network Approach}

The finite network approach bases the model on a physical level and tries to put the spatial dependency of the physical fields into network elements. Since we focus on acoustic transducers, we have to deal with electric, mechanical and acoustic systems and need analogies in order to achieve an appropriate electric network. In this sense, we define the quantities as listed in Tab. 1 (complex quantities are marked by an underline). To achieve at an electric network, we will use the $\mathrm{pU}$ and

Table 1: Mechanical, electrical and acoustic networks

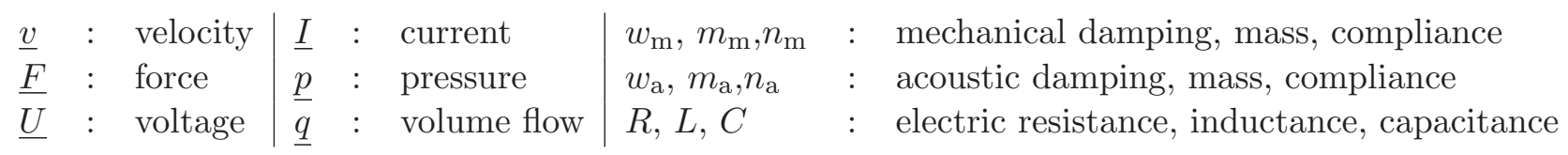

\begin{tabular}{l|llllll} 
Network & $\begin{array}{l}\text { Potential } \\
\text { quantity }\end{array}$ & $\begin{array}{l}\text { Flow } \\
\text { quantity }\end{array}$ & Impedance & Passive elements \\
\hline Mechanical & $\underline{v}$ & $\underline{F}$ & $\underline{Z}_{\mathrm{m}}=\underline{F} / \underline{v}$ & $\underline{Z}_{\mathrm{mw}}=w_{\mathrm{m}}$ & $\underline{Z}_{\mathrm{mk}}=1 /\left(j \omega n_{\mathrm{m}}\right)$ & $\underline{Z}_{\mathrm{mm}}=j \omega m_{\mathrm{m}}$ \\
Electrical & $\underline{U}$ & $\underline{I}$ & $\underline{Z}_{\mathrm{e}}=\underline{U} / \underline{I}$ & $\underline{Z}_{\mathrm{eR}}=R$ & $\underline{Z}_{\mathrm{eL}}=j \omega L$ & $\underline{Z}_{\mathrm{eC}}=1 /(j \omega C)$ \\
Acoustic & $\underline{p}$ & $\underline{q}$ & $\underline{Z}_{\mathrm{a}}=\underline{p} / \underline{q}$ & $\underline{Z}_{\mathrm{aw}}=w_{\mathrm{a}}$ & $\underline{Z}_{\mathrm{am}}=j \omega m_{\mathrm{a}}$ & $\underline{Z}_{\mathrm{ak}}=1 /\left(j \omega n_{\mathrm{a}}\right)$
\end{tabular}

FU analogy. Furthermore, we transform the mechanical elements to their corresponding acoustic elements by $\underline{Z}_{\mathrm{a}}=\underline{Z}_{\mathrm{m}} / A^{2}$ with $A$ the appropriate cross section [7]. Therewith, the next step will be to deriving a two-port equivalent circuit, which converts the acoustic quantities pressure and volume flow to the electrical ones voltage and current. Linearizing the relations at the operating point, which is defined by the electrical pre-stressing with $U_{0}[1]$

$$
\begin{aligned}
\underline{i}+j \omega C_{0} \underline{u} & =\underline{i}_{\mathrm{T}}=\frac{\varepsilon_{0} U_{0}}{x_{*}{ }^{2}} \underline{q}=-\frac{1}{\Gamma} \underline{q} \\
\underline{p}-\frac{1}{j \omega C_{\mathrm{aM}}^{*}} \underline{q} & =\underline{p}_{\mathrm{T}}=-\frac{1}{\Gamma} \underline{u}
\end{aligned}
$$

and the equivalent circuit is displayed in Fig. 2. The individual elements compute by

$$
C_{0}=\frac{\varepsilon_{0} A}{x_{*}} ; \quad \Gamma=\frac{x_{*}{ }^{2}}{\varepsilon_{0} U_{0}} ; \quad \frac{1}{C_{\mathrm{M}}^{*}}=\frac{1}{n_{\mathrm{aM}}^{*}}=\frac{1}{n_{\mathrm{aM}}}-\frac{1}{\Gamma^{2} C_{0}},
$$

where $x_{*}$ is the air-gap at the operating point (electrical pre-stressing with $U_{0}$ ), $A$ the electrode surface, $\varepsilon_{0}$ the permittivity of vacuum, $C_{0}$ the electric capacitance at $x_{*}, n_{\text {aM }}$ the mechanical compliance of the membrane already transformed to the acoustic side and $\Gamma$ the transducer constant. In a last step, we plug this equivalent circuit into the overall acoustic network for the transducer as displayed in Fig. 3. All the electric resistances, inductances and capacitances model acoustic and mechanical damping, mass and stiffness. Starting at the sound source, we model:

- the opening of the housing by an acoustic damping $R_{\text {in }}$ and mass $L_{\text {in }}$ 


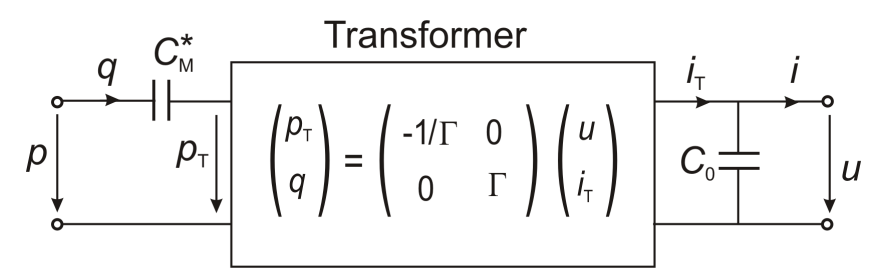

Figure 2: Linear equivalent circuit for the electrostatic transducer.



Figure 3: Acoustic network including the electrostatic transducer (linearized at the working point).

- the acoustic stiffness of the cavity between opening of the housing and membrane by $C_{\mathrm{in}}$

- the acoustic radiation of the membrane by $R_{\mathrm{rad}}$ and $L_{\mathrm{rad}}$

- the mass and stiffness (including the electrostatic softening) by $L_{\mathrm{M}}$ and $C_{\mathrm{M}}^{*}$

- the hole for pressure balance by $R_{\mathrm{vent}}$ and $L_{\mathrm{vent}}$

- the compliance of the air-gap by $C_{\text {gap }}$

- the acoustic flow in the air-gap from the membrane to the backplate holes and through them by $R_{\text {gap }}, R_{\mathrm{bp}}$ and $L_{\mathrm{bp}}$

- the compliance of the back volume $C_{\mathrm{V}}$.

Therewith, we have achieved an overall model of the silicon microphone, and we can quickly check, if the design goals can be roughly achieved. However, realistic values for the individual elements are missing, which could be obtain by measurements. The problem is just that at this stage of the development process, we do not have a prototype and so the most appropriate way is to use the FE method, which accurately solves the underlying PDEs. As an example, we demonstrate in Fig. 4 the results of compliance computations of advanced membrane designs with corrugations, which minimizes the residual stress due to fabrication. Since we just consider one-dimensional deformations in finite network models, we compute the mechanical compliance $n_{\mathrm{m}}$ of the corrugated membrane from the FE results as follows

$$
n_{\mathrm{m}}=\frac{\iint w(x, y) d x d y}{F_{\mathrm{p}}} .
$$




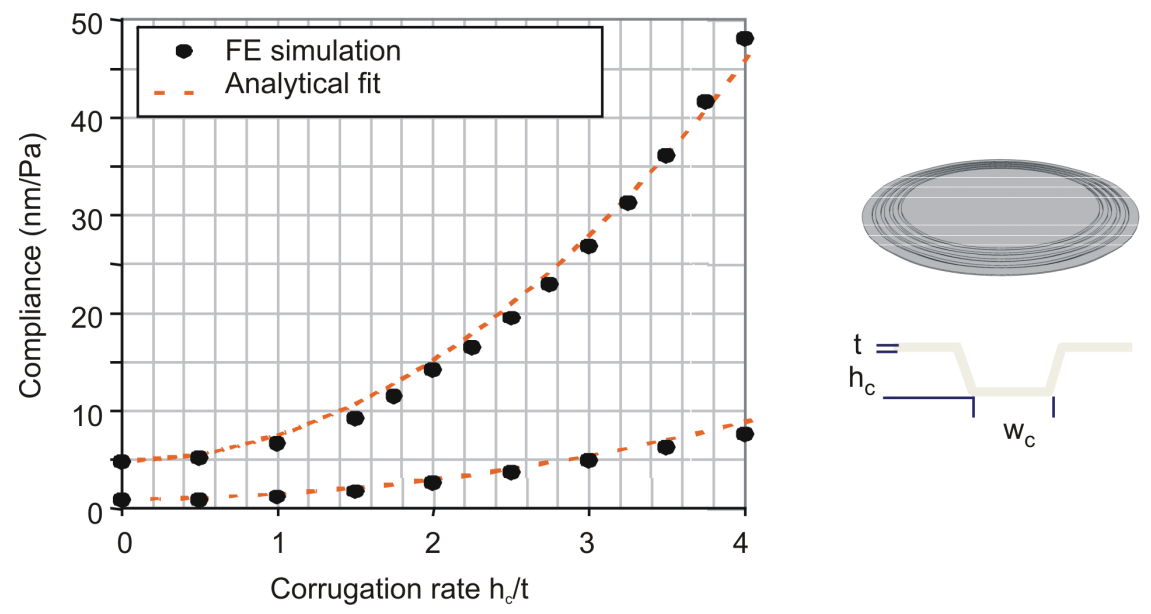

Figure 4: Computed membrane compliance over corrugation rate.

In (4) $w(x, y)$ is the mechanical deflection and $F_{\mathrm{p}}$ the applied pressure force. To fit an analytical formula for $n_{\mathrm{m}}$ used in the finite network approach, we perform this computations for different corrugation rates $h_{\mathrm{c}} / t$ and apply a standard least squares optimization.

\section{$3 \quad$ Finite Element Approach}

The challenges for FE based simulations of acoustic electrostatic-mechanical transducers as the silicon microphone can be summarized as follows. First, one has to deal with three physical fields, the acoustic, the electrostatic and mechanical field. Secondly, the coupling mechanisms between the physical fields have to be taken into account, which are for the electrostatic-mechanical one highly nonlinear. Due to the electrostatic force, the mechanical structure is subjected both to rigid motions and elastic deformations. Furthermore, the change on the structure (air-gap) in turn strongly influences the electric field and thus the electrostatic force distribution. Using predictor values for the electric potential in order to compute the electrostatic force and predictor values for the mechanical displacement to update the configuration for the computation of the electrostatic field, we can split the coupled system of partial differential equations into a mechanical part and an electric part. To guarantee a full coupling between the two fields, we perform an iterative solution process. The convergence test is based on the following stopping criterion[4]

$$
\frac{\left\|\mathbf{u}_{k+1}^{n+1}-\mathbf{u}_{k}^{n+1}\right\|_{2}}{\left\|\mathbf{u}_{k+1}^{n+1}\right\|_{2}}<\delta_{\mathrm{o}},
$$

with $\mathbf{u}$ the nodal vector of mechanical displacements, $\delta_{\mathrm{o}}$ an adjustable accuracy (in practice $10^{-2}-10^{-3}$ ), \|\|$_{2}$ the $\mathrm{L}_{2}$-norm, $k$ the iteration counter for the outer loop (electrostatic-mechanical iteration) and $n$ the time step number. For the successful application of this scheme, we need an accurate method for the electrostatic force computation and an efficient treatment of the moving/deforming body problem.

- Computation of electrostatic force:

The electrostatic force acting on the mechanical structure is computed via the virtual work principle. Therewith, the electrostatic force $\mathbf{F}_{\text {elec }}$ in the direction of a virtual displacement $\delta \mathbf{r}$ computes by the virtual change of the electrostatic energy $\delta W_{\text {elec }}$ according to

$$
\delta W_{\text {elec }}=\mathbf{F}_{\text {elec }} \cdot \delta \mathbf{r} .
$$


We have extended the method given in [2] for the computation of the local force distribution acting on electrically charged mechanical structures within an electrostatic field. For details we refer to [4].

- Treatment of a moving body in an electric field:

Due to a movement and/or deformation of the mechanical structures in electrostatic devices, the finite elements for computing the electrostatic field will be deformed, and thus will cause numerical inaccuracy. These deformations have to be controlled, and a re-meshing of the simulation domain has to be performed before an intersection of finite elements occurs. To avoid this problem, we have developed a smoothing mesh technique. We apply the mechanical deformations of the structures as Dirichlet boundary conditions in a subsequent pseudomechanical computation such that all finite elements within the electrostatic computational domain (in our case the region between the deformable electrode and the back electrode) gets somehow uniformly deformed. This is displayed in Fig. 5 for subsequent time steps, in which the deformation of the mechanical structure strongly increases. As lon as no contact problems are considered, this method is efficient, since no re-meshing is necessary.

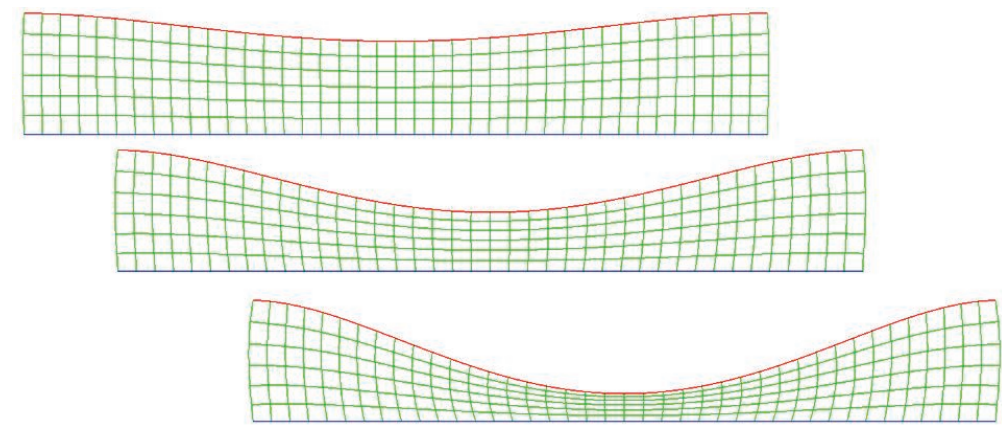

Figure 5: Moving mesh technique.

The described computational scheme has been implemented in the FE software CFS++[5], and successfully applied to many practical applications. Here, we will apply it for the computation of the total harmonic distortion (THD) of the silicon microphone, which calculates as

$$
T H D=\frac{\sqrt{\sum_{i=1}^{\infty} S_{i}^{2}}}{\sqrt{\sum_{i=0}^{\infty} S_{i}^{2}}}
$$

with $S_{i}$ the $i$-th higher harmonic of the receiving signal $S$. The THD is the limiting factor for the use of micromachined microphones at high pressure levels. The membranes used in such microphones are deflected strongly, and show a nonlinear mechanical behavior. Furthermore, the nonlinearity due to the electrostatic force accounts to the THD. We have simulated the dynamic reception signals of the silicon microphone with corrugated membranes (see. Fig. 6(a)) excited by a $114 \mathrm{~dB}$ SPL signal at $1 \mathrm{kHz}$. The measured output voltage of the microphone (electric circuit with DC-biasing voltage source and preamplifier) is direct proportional to the change of the electric capacitance. Therewith, we have performed a transient simulation and have computed the THD from the obtained capacitance. The comparison of measured and computed THD values are listed in Tab. 2, and Fig. 6(b) displays the frequency spectrum of the capacitance change for design M1. This clearly demonstrates, that for such investigations, an advanced computational scheme solving the PDEs with their couplings is indispensable. 


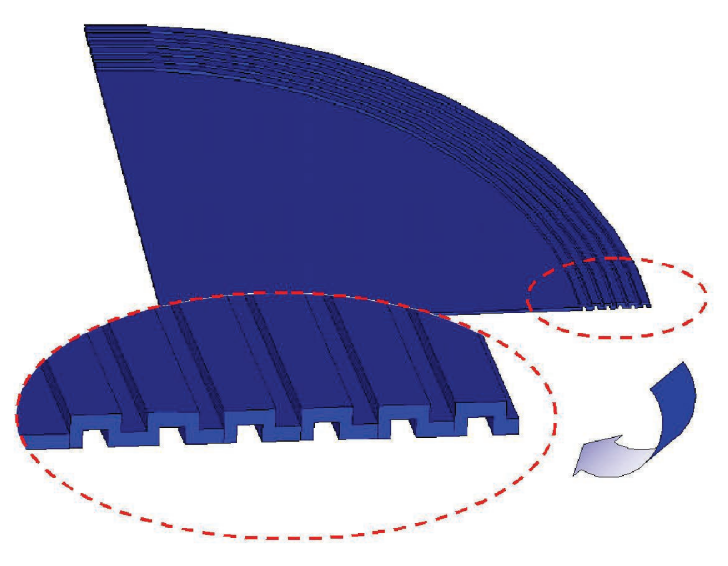

(a) Corrugated membrane



(b) Frequency spectrum of normalized change in capacitance for the microphone with membrane M1

Figure 6: Corrugated membrane and frequency spectrum of the capacitance change

Table 2: Total Harmonic Distortion (THD) of silicon microphones with different membranes M1-M4 for $\mathrm{SPL}=114 \mathrm{~dB}$ at $1 \mathrm{kHz}$

\begin{tabular}{c||c|c|c|c} 
& M1 & M2 & M3 & M4 \\
\hline \hline measured & $13.6 \%$ & $2.1 \%$ & $1.7 \%$ & $1.4 \%$ \\
simulated & $12.6 \%$ & $2.7 \%$ & $2.3 \%$ & $1.6 \%$
\end{tabular}

\section{References}

[1] R. Ballas, G. Pfeifer, and R. Werthschützky. Elektromechanische Systeme der Mikrotechnik und Mechatronik. Springer, 2009.

[2] J. Coulomb and G. Meunier. Finite Element Implementation of Virtual Work Principle for Magnetic and Electric Force and Torque Computation. IEEE Trans. on Magnetics, 20(5):1894$1896,1984$.

[3] M. Füldner. Modellierung und Herstellung kapazitiver Mikrofone in BiCMOS-Technologie. PhD thesis, University of Erlangen-Nuremberg, 2004.

[4] M. Kaltenbacher. Numerical Simulation of Mechatronic Sensors and Actuators. Springer, Berlin, 2. edition, 2007. ISBN: 978-3-540-71359-3.

[5] M. Kaltenbacher. Advanced simulation tool for the design of sensors and actuators. In Proc. Eurosensors XXIV. Elsevier, 2010. CD-ROM-Proceedings, Linz.

[6] S. J. Rupitsch and R. Lerch. Inverse method to estimate material parameters for piezoceramic disc actuators. Applied Physics A, 97(4)::735-740, 2009.

[7] M. Zollner and W. Zwicker. Elektroakustik. Springer, 1993. 